ORIGINAL PAPER

\title{
Clinical Significance of PON1 L55M, Q192R AND I102V POLYMORPHISMS AND THEIR ASSOCIATION WITH PROSTATE CANCER RISK IN POLISH MEN
}

\author{
Marta Heise $^{1}$, Piotr Jarzemski ${ }^{2}$, Aneta Bąk ${ }^{1}$, Anna Junkiert-Czarnecka ${ }^{1}$, \\ Maria Pilarska-Deltow ${ }^{1}$, Maciej Borysiak ${ }^{2}$, Olga Haus ${ }^{1}$
}

\author{
${ }^{1}$ Department of Clinical Genetics, Faculty of Medicine, Collegium Medicum in Bydgoszcz, Nicolaus Copernicus University \\ in Torun, Poland \\ ${ }^{2}$ Department of Urology, Jan Biziel University Hospital in Bydgoszcz, Poland
}

\begin{abstract}
We tested the association between PON1 L55M, Q192R and I102V polymorphic variants and PC risk in Polish men. DNA from 110 consecutive, newly diagnosed patients hospitalized because of PC and DNA from 110 men - volunteers, healthy at the time of the study. PCR-RFLP method. In our study the average age at PC diagnosis of 55MM genotype carriers from families fulfilling Hereditary Prostate Criteria (HPC) was statistically significantly lower (by 7 years) than the average age at the disease diagnosis of 55MM carriers from families without HPC (54.6 \pm 6.7 vs. $61.9 \pm 5.4$, respectively, $\mathrm{p}=0.03$ ). The probability of 5 -year survival for the $55 \mathrm{MM}$ carriers was $81.3 \%$, compared to $95.7 \%$ for non-carriers $(\mathrm{p}=0.08$, tendency). This is the first study in Polish men evaluating the impact of PON1 genetic polymorphisms on prostate cancer development and its clinical course. PON1 $55 \mathrm{MM}$ variant may be probably associated with younger age at PC onset in men from families with HPC and with a shorter survival. However, more extensive studies on a larger number of PC patients, possibly from various populations, are necessary to confirm, and extend our findings.
\end{abstract}

Key words: prostate cancer; PON1 L55M, Q192R and I102V polymorphisms, predisposition, clinical characteristics.

\section{Introduction}

A cancer has become a major public health problem and global burden because of the high morbidity and mortality rate. According to the American Cancer Society, in 2017, prostate cancer (PC) was the commonest diagnosed malignancy and the third leading cause of cancer mortality in men in the US [1]. There were 161360 new diagnoses of prostate cancer and 26730 deaths from the disease $[1,2]$. Genetic basis of prostate cancer is very complex. Thus, identification of PC high predisposition genes, as well as prevention or early diagnosis of the disease are extremely urgent. Some studies show that BRCA1, BRCA2, CHEK2, NBS1 or HOXB13 gene mutations which play an important role in cell cycle regulation, DNA repair and embryonic development of various organs are associated with higher risk of PC but none of these is a high risk gene for prostate cancer development. Literature analysis indicates that redox 
signaling plays an important role in malignant tumor cell transformation through reactive oxygen species (ROS) mediated function. Dysregulation of this redox balance is implicated in the development of various diseases including cancer $[3,4,5,6,7,8,9,10$, $11,12,13,14,15]$.

An antioxidant paraoxonase 1 (PON1) is an important endogenous free radical scavenger which plays a role in preventing the effects of systemic oxidative stress which is defined as a redox imbalance between prooxidant and antioxidant systems with either an overproduction of oxidants or a deficiency of antioxidant agents [16]. Under oxidative stress, excess ROS can cause damage to many cellular and extracellular constituents, including DNA, proteins and lipids [17]. The physiological role of PON1 protein is not exactly known, however increasing evidence indicates that it can assure protection against oxidative damage by hydrolysing lipid hydroperoxides and by protecting the low density lipoproteins from oxidative modifications [18].

The human PON1 protein is coded by PON1 gene located on the long arm of chromosome 7 (7q21.3). Its the most common polymorphisms localize in the coding region: a leucine $(\mathrm{L})$ to methionine $(\mathrm{M})$ substitution at position 55 (L55M, p.Leu55Met, c.163T > A) and a glutamine $(\mathrm{Q})$ to arginine $(\mathrm{R})$ substitution at position 192 (Q192R, p.Gln192Arg, c.575A>G). The L55M [rs854560] and Q192R [rs662] polymorphisms affect paraoxonase 1 (PON1) serum concentration and activity of PON1 gene [19, 20, 21]. It was suggested that PON1 55M allele could correlate with higher PON1 activity than PON1 55L allele. In another study, it was observed that the differences in the PON1 serum levels linked to the $\mathrm{L} 55 \mathrm{M}$ polymorphism were due to the reduced stability of the PON1 55M protein [22]. The hydrolyzing activity of the protein encoded by the 192RR, second the most common genotype of PON1 gene, is 8-fold higher than that encoded by the 192QQ. This polymorphism also affects the serum protein concentration. Homozygous 192RR individuals have higher enzyme concentrations than the homozygous 192QQ individuals [15, 23, 24]. Additionally, Marchesani et al. detected new polymorphic variant in exon 4 , an $A \rightarrow G$ substitution at nucleotide position 304 resulting in an isoleucine $\rightarrow$ valine substitution at codon 102 of the PON1 (I102V, p.Ile102Val). The authors indicated that PON1 I102V is associated with decreased serum paraoxonase stability [25].

Variations due to the presence of single nucleotide polymorphisms in antioxidant enzyme genes may contribute to the interindividual differences of transcript levels and enzyme activities, which might play a significant role in the prostate cancer development $[14,22]$. Therefore, the aim of the present study was to investigate the relation between PON1 L55M, Q192R and I102V polymorphisms and prostate can- cer risk in Polish men. Additionally, we measured the impact of these variants and their constructed haplotypes on clinical course of the disease, including overall survival time.

\section{Material and methods}

The material of the investigations were archival DNA samples stored in the Department of Clinical Genetics CM UMK in Bydgoszcz from 2005 to 2007. DNA was isolated from EDTA anticoagulated peripheral blood of 110 consecutive, newly diagnosed prostate cancer (PC) patients, from all over Poland, regardless of age at PC onset, family history and histological type of cancer. They were hospitalized because of PC at Department of Urology of the J. Biziel University Hospital in Bydgoszcz. The age at PC diagnosis ranged from 45 to 75 years (the mean age $59.9 \pm 5.9$ ). Family history was analyzed either by the construction of a family pedigree or the completion of a standardized questionnaire by patients. All cases of first- and second- degree relatives diagnosed with prostate cancer and their age at disease diagnosis were recorded. The estimation of patient families as those with a history suggesting hereditary risk of prostate cancer (HPC) was performed on the basis of criteria defined by Carter et al. [26] and Cybulski et al. [27]. Among 110 prostate cancer patients, 25 $(22.7 \%)$ originated from families suspected of Hereditary Prostate Cancer (Fig. 1).

Information about the PSA level before the surgical operation was available for 97 patients, about grade of prostate cancer for 101 patients, and about tumor

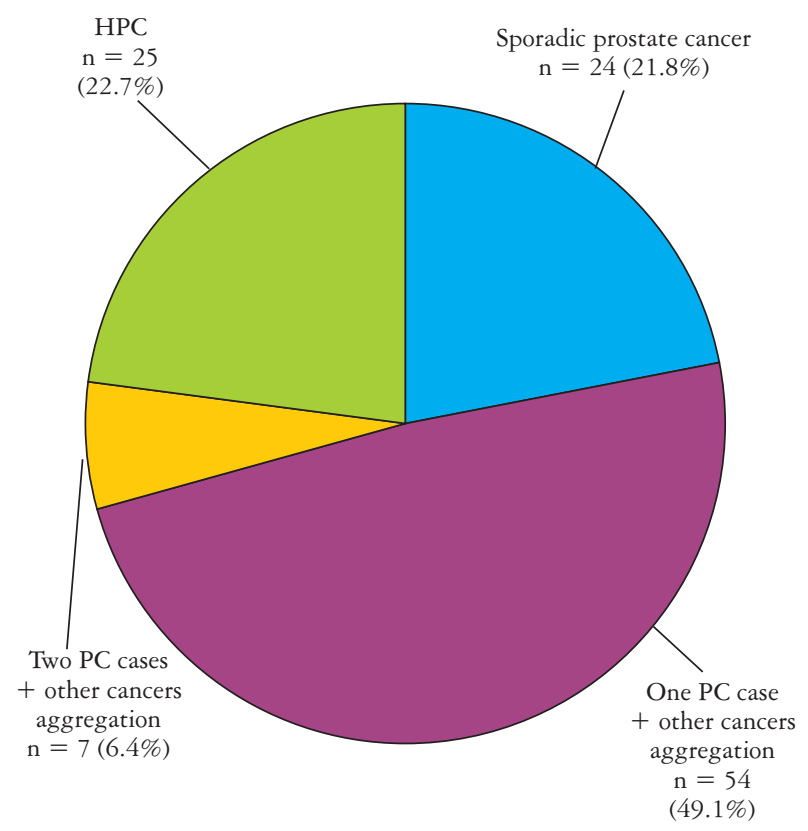

Fig. 1. Families classification depending on the number of PC cases 
stage for 102 patients. Data on survival were available for 103 PC patients.

In 61 of $110(55.45 \%)$ families, an aggregation of cancers of the breast, stomach, colon, ovary, lung, larynx, bladder and kidney, as well as melanoma was present, in addition to prostate cancer.

Control group consisted of DNA samples isolated from peripheral blood of 110 men, volunteers, healthy at the time of the investigations, i.e. (without prostate cancer on the basis of PSA concentration and/or digital rectal examination - DRE). The medical examinations were performed in them as a part of PC prophylaxis. Control molecular test was offered to all men with correct result of DRE and with a normal age-specific value of PSA. The age of men from control group ranged from 46 to 74 years (the mean age was 59.9 \pm 6.6 ) and matched to the PC group.

The study protocol was approved by the Ethics Committee of the Collegium Medicum Nicolaus Copernicus University in Bydgoszcz, Poland. Every PC patient and a men from control group gave their written informed consent for the use of their DNA sample for genetic testing.

\section{Polymorphisms analysis}

Genomic DNA was isolated from peripheral blood leukocytes by the standard salting-out method. The PCR-RFLP was used for determining the L55M and Q192R polymorphisms, and Sanger sequencing method for determining the I102V. The primers for amplification of coding regions including L55M, Q192R and I102V were designed using Primer3 software (http://frodo.wi.mit.edu/).

The PCR amplification was done for the fragments of PON1 gene with following pairs of primers: Forward (F): 5' - GAAGAGTGATGTATAGCCCCA G - 3' together with Reverse (R): 5' - TTTAATCCAGAGCTAATGAAAGCC - 3' for determination of the $\mathrm{L} 55 \mathrm{M}$ polymorphism and F: 5' - TATTGTTGCTGTGGGACCTGA G - 3' together with R: 5' - CACGCTAAACCCAAATACATCTC - 3' for determination of the Q192R polymorphism. The conditions and components of PCR reaction are available on request. The restriction enzyme N1aIII (Biolabs) was used for determination of the L55M. Digestion with NlaIII generated the following fragments: for $55 \mathrm{M}$ allele fragments of $127 \mathrm{bp}$ and $42 \mathrm{bp}$, for 55L allele a single fragment of $169 \mathrm{bp}$. The Alw1 (Biolabs) restriction enzyme was used to identify the possible Q192R genotypes. The Alw1 digestion generated the following fragments: for 192R allele fragments of $63 \mathrm{bp}$ and $36 \mathrm{bp}$ and for 192Q allele - a single fragment of $99 \mathrm{bp}$. The digested PCR products of the two PON1 polymorphisms were separated on $3 \%$ agarose gel and visualized using Midori Green DNA staining.
The I102V polymorphism was genotyped using Sanger sequencing. The primers for amplification of PON1 coding region were: 5' - CTTGGATGGAGCCAAGGATA - 3' (F) and 5' - CCTCACCAGCTCCAAGTCTC - 3' (R). The amplified DNA amplicon was sequenced using BigDye terminator v3.1 Cycle Sequencing Kit (Life Technologies), according to manufacturer protocol. Sequencing products were analyzed on the ABI prism 3130 Genetic Analyzer (Applied Biosystems, Life Technologies). All sequences were compared with the PON1 RefSeq sequence (NG_008779) for I102V polymorphism detection. Sequencing analysis of the coding region of PON1 was performed in all men from study and control groups.

\section{Statistical analysis}

The polymorphisms frequencies in prostate cancer patients and controls were compared. To evaluate the effects of three Single Nucleotide Polymorphisms (SNPs) on prostate cancer risk, haplotypes were constructed. Haplotypes frequencies were estimated using statistical software. Odds ratios (ORs) with 95\% confidence intervals (CIs) were used to assess the strength of the associations. ORs were generated from two-by-two tables and statistical significance was assessed with the Fisher's exact test or the chisquare test when appropriate. Mean age at PC diagnosis was compared between polymorphism carriers and non-carriers using Student's t-test. For the survival analysis, the patients were followed from the date of biopsy (confirmation of prostate cancer) until death, if applicable or the end of observations. In living patients five year survival was analyzed. The vital status and the date of death were requested from the Polish Ministry of Health. The Kaplan-Meier curves for the probability of survival of PON1 polymorphic variant carriers and non-carriers have been estimated.

\section{Results}

The comparison of the PON1 L55M and Q192R genotypes and haplotypes frequencies between prostate cancer patients and healthy controls were shown in Table I and Table II. The comparison of the both groups of men did not show any statistically significant differences or trends in genotypes and haplotypes distribution. However, the 55MM homozygous genotype was detected in $14.5 \%$ of PC patients vs $17.3 \%$ of controls ( $p=0.58, \mathrm{NS}$ ) and the 192RR homozygous genotype was detected in $5.5 \%$ of PC patients vs. $4.6 \%$ of controls ( $p=0.77, \mathrm{NS}$ ). The PON1 55LM+192QQ haplotype was the most frequently found in both prostate cancer and control groups (26.6\% and $26.9 \%$, respectively). The PON1 $55 \mathrm{MM}+192 \mathrm{RR}$ haplotype was detected in no man. A PON1 102II wild type homozygous genotype was 
Table I. The frequency of two L55M and Q192R polymorphisms and PC risk

\begin{tabular}{lccccc}
\hline PON1 L55M & $\begin{array}{c}\text { PC Patients } \\
\mathbf{N}=110(\%)\end{array}$ & $\begin{array}{c}\text { Controls } \\
\mathbf{N}=110(\%)\end{array}$ & OR & P-VAlue & 95\% CI \\
\hline LL & $40(36.4)$ & $42(38.2)$ & 0.93 & 0.78 & $0.54-1.60$ \\
\hline LM & $54(49.1)$ & $49(44.5)$ & 1.20 & 0.50 & $0.71-2.04$ \\
\hline MM & $16(14.5)$ & $19(17.3)$ & 0.82 & 0.58 & $0.39-1.68$ \\
\hline LL or LM & $94(85.5)$ & $91(82.7)$ & 1.23 & 0.58 & $0.59-2.53$ \\
\hline PON1 Q192R & PC PATIENTS & ConTROLS & OR & P-VALUE & $95 \%$ CI \\
\hline QQ & $59(54.1)$ & $62(57.4)$ & 0.88 & 0.63 & $0.51-1.50$ \\
\hline QR & $44(40.4)$ & $41(38.0)$ & 1.11 & 0.72 & $0.64-1.91$ \\
\hline RR & $6(5.5)$ & $5(4.6)$ & 1.20 & 0.77 & $0.36-4.06$ \\
\hline QQ or QR & $103(94.5)$ & $103(95.4)$ & 0.83 & 0.77 & $0.25-2.82$ \\
\hline OR - odds ratio; $p \leq 0.05$ & & & & &
\end{tabular}

Table II. The frequency of two L55M and Q192R haplotypes and PC risk

\begin{tabular}{|c|c|c|c|c|c|c|}
\hline $\begin{array}{l}\text { PON1 } \\
\text { L55M }\end{array}$ & & $\begin{array}{l}\text { PC PATIENTS } \\
\mathrm{N}=110(\%)\end{array}$ & $\begin{array}{c}\text { Controls } \\
\mathrm{N}=110(\%)\end{array}$ & OR & P-VALUE & $95 \% \mathrm{CI}$ \\
\hline $\mathrm{L} 55 \mathrm{M}$ & Q192R & & & & & \\
\hline \multirow[t]{3}{*}{ LL } & QQ & $16 / 109(14.7)$ & $16 / 108(148)$ & 0.99 & 0.98 & $0.47-2.10$ \\
\hline & QR & $18 / 109(16.5)$ & 20/108 (18.5) & 0.87 & 0.70 & $0.43-1.75$ \\
\hline & $\mathrm{RR}$ & $5 / 109(4.6)$ & $5 / 108(4.6)$ & 0.99 & 0.99 & $0.28-3.52$ \\
\hline \multirow[t]{3}{*}{ LM } & QQ & 29/109 (26.6) & 29/108 (26.9) & 0.99 & 0.97 & $0.54-1.80$ \\
\hline & QR & $24 / 109(22.0)$ & 20/108 (18.5) & 1.24 & 0.52 & $0.64-2.41$ \\
\hline & $\mathrm{RR}$ & $1 / 109(0.9)$ & 0/108 (0.0) & 3.00 & 0.50 & $0.12-74.46$ \\
\hline \multirow[t]{3}{*}{ MM } & QQ & $14 / 109(12.8)$ & $17 / 108(15.7)$ & 0.79 & 0.54 & $0.37-1.69$ \\
\hline & QR & 2/109 (1.8) & 1/108 (0.9) & 2.00 & 0.57 & $0.18-22.39$ \\
\hline & $\mathrm{RR}$ & $0 / 109(0.0)$ & 0/108 (0.0) & - & - & - \\
\hline
\end{tabular}

$O R$ - odds ratio; $p \leq 0.05$

detected in all PC patients and in all men from control group, therefore, in further analyzes it was omitted.

We observed almost 2-fold higher 55MM genotype frequency in PC patients from families with HPC compared to its frequency in patients from families without HPC (20.0\% vs. $12.9 \%)$. The difference was not statistically significant $(\mathrm{p}=0.38)$. However, the average age at PC diagnosis of 55MM genotype carriers from families fulfilling HPC criteria was statistically significantly lower (by 7 years) than the average age at the disease diagnosis of $55 \mathrm{MM}$ carriers from families without HPC (54.6 \pm 6.7 vs. 61.9 \pm 5.4 , respectively, $\mathrm{p}=0.03$ ).

The age at PC diagnosis of the 192RR carrier (66 years) from a family with sporadic prostate cancer was statistically significantly older than the average age at PC diagnosis of $192 \mathrm{RR}$ carriers (56.3 \pm 1.7$)$ from families with one PC case and other cancers aggregation. The difference was statistically significant $(p=0.01)$, however, it was calculated on the basis of the age at PC diagnosis of only one man with 192 RR.

Data on survival were available for 103 investigated PC men. There were 3 deaths recorded among sixteen 55MM homozygous genotype carriers and six deaths among 87 non-carriers. The probability of 5-year survival for the $55 \mathrm{MM}$ carriers was $81.3 \%$, compared to $95.7 \%$ for non-carriers ( $\mathrm{p}=0.08$, tendency). Figure 2 shows the Kaplan-Meier curves for these PON1 genotype carriers.

The probability of 5-year survival for the 192RR carriers was $100 \%$, compared to $93.2 \%$ for carriers of other genotypes ( $\mathrm{p}=0.44, \mathrm{NS})$. The Kaplan-Meier curves for PON1 Q192R prostate cancer patients are presented on Fig. 3.

In the present study, both L55M and Q192R polymorphisms genotype frequencies were not related to 


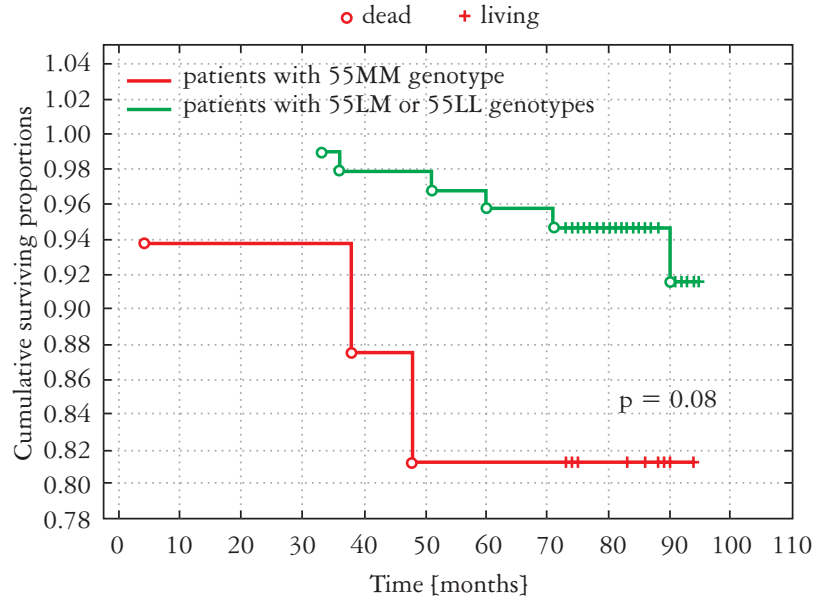

Fig. 2. The Kaplan-Meier probability curves for overall survival from PC diagnosis for patients with 55MM genotype compared to patients with $55 \mathrm{LM}$ or $55 \mathrm{LL}$. The red line represents the survival for the $55 \mathrm{MM}$ carriers and the green line the survival for $55 \mathrm{LM}$ or $55 \mathrm{LL}$ carriers

the patients age. We did not observed a statistically significant difference between the average age at PC onset of $55 \mathrm{MM}$ genotype carriers compared to the average age at PC onset of 55LM and 55LL genotypes carriers $(59.6 \pm 6.6$ vs. $60.0 \pm 5.8$, respectively, $\mathrm{p}=0.84)$. Similar results were obtain for the analysis of the average age at PC onset of 192RR genotype carriers compared to $192 \mathrm{QR}$ and $192 \mathrm{QQ}$ genotypes carriers ( $59.7 \pm 5.5$ vs. $59.9 \pm 5.6$, respectively, $\mathrm{p}=0.92)$. The 192RR genotype was detected in $4(3.7 \%)$ PC men and in one $(0.9 \%)$ man from the control group between 51 and 60 years of age $(\mathrm{OR}=$ $3.78, \mathrm{p}=0.24$, NS).

In $18.8 \%$ of PC men, $55 \mathrm{MM}$ carriers, prostate cancer cells were characterized by a low degree of differentiation ( $\mathrm{SG} \geq 8$ ), compared to $10.3 \%$ of men with 55LL genotype and $11.3 \%$ of men with 55LM genotype. Moreover, the cancers showed a high level of progression at the time of diagnosis (T3/4) in $31.3 \%$ of men with $55 \mathrm{MM}$ (in $10 \%$ of men with $55 \mathrm{LL}$ and in $15.1 \%$ of men with $55 \mathrm{LM}$ ). $15.4 \%$ of men with $55 \mathrm{MM}$ had preoperative PSA $>20 \mathrm{ng} / \mathrm{ml}$ compared to $7.9 \%$ of men with 55LL and $5.7 \%$ of men with 55LM. However, all above differences were no statistically significant. No statistically significant differences in the presence of PSA concentration $>20 \mathrm{ng} / \mathrm{ml}$, high grade Gleason Score (GS $\geq 8$ ) or advanced TNM stage (T3/4), depending on Q192R carrier status, were also observed.

\section{Discussion}

This is the first study in Polish men evaluating the impact of PON1 genetic polymorphisms on prostate cancer development and its clinical course. The oxidative stress has a strong impact on cancer formation

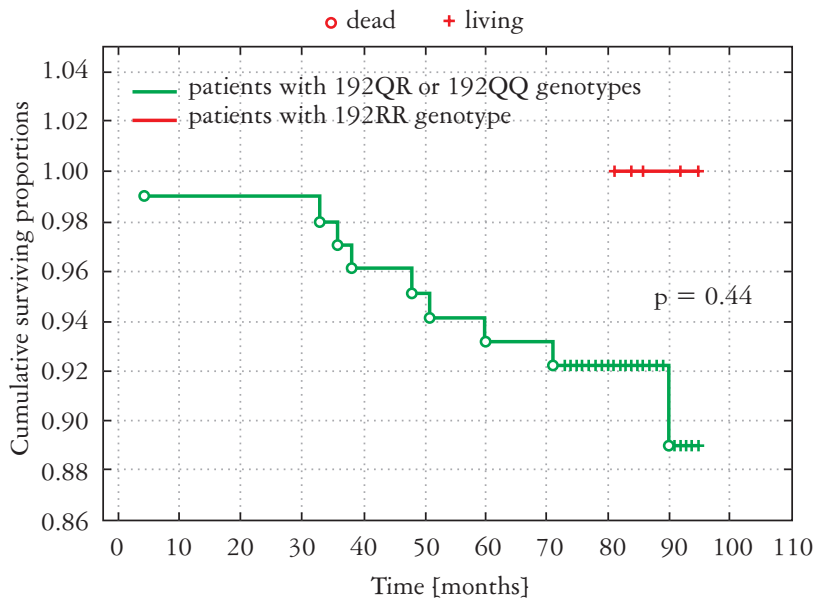

Fig. 3. The Kaplan-Meier probability curves for overall survival from PC diagnosis for patients with 192RR genotype compared to patients with 192QR or 192QQ. The red line represents the survival for the 192RR carriers and the green line the survival for 192QR or 192QQ carriers

and development. However, the human body has several protective systems with an ability to remove harmful free radicals. In the present study we did not find any statistically significant relationship of the L55M and Q192R genotypes and their constructed haplotypes with PC, however, some observations are worth noting. In contrast to our results, Antognelli et al. found a relationship between PON1 55LM-MM and 192QR polymorphisms and PC risk in the Italian population [28]. Fang et al. performed a meta-analysis based on 25 studies and found association between $55 \mathrm{LM}$ heterozygous genotype compared to $55 \mathrm{LL}$ wild type genotype with increased prostate cancer risk [29]. Zhang et al. analyzed 8112 cases and 10037 controls from 32 published case-control studies to assess the connection between PON1 192R allele with many types of cancers including prostate cancer. They showed that 192QR compared to 192QQ and 192RR+QR compared to 192QQ were associated with an increased $\mathrm{PC}$ risk $(\mathrm{OR}=1.782$, $\mathrm{p}=0.000$ and $\mathrm{OR}=1.281, \mathrm{p}=0.056$, respectively) [30]. In another meta-analysis, based on 21 studies, Chen $e t$ al. indicated that PON1 L55M was a risk factor for prostate cancer in the heterozygote model (55LM vs. 55LL: $\mathrm{OR}=1.304, \mathrm{p}=0.067$ ) [31].

In the present study, no relationship was observed for I102V with prostate cancer. The 102II homozygous genotype was detected in all PC patients and in all men from control group. Marchesani et al. observed that men who carry the PON1 102V allele compared to non-carriers have more than 6-fold higher increased relative risk for the PC first clinical manifestation. In an independent analysis also performed by Marchesani et al. the I102V was 4.3-fold more frequent in group of 69 men with familial prostate cancer compared to 69 healthy control subjects. 
Additionally, these authors indicated that L55M or Q192R were not associated with PC [25]. The size of prostate cancer increased risk associated with the I102V may be different in Polish and Finnish men. Comparing to Polish ethnically healthy homogenous population, Finns have a relatively small founder population and many immigrants from other countries, compared to Polish population.

In the present study, the 55MM frequency was higher in men from HPC families than in men from families without HPC (20\% vs. $12.9 \%$, NS). The average age at PC diagnosis was statistically significantly younger (by 7 years) in 55MM carriers from HPC families than those from families without HPC. Thus, the carrying of $55 \mathrm{MM}$ may be associated with hereditary prostate cancer and a younger age at PC onset, however, in order to make unambiguous conclusions, more studies are needed, on larger groups of patients. The 192 RR frequency was similar in men from HPC families and those from families without HPC (4\% vs. 6\%). This indicates that there is no relationship between PON1 192 RR genotype and hereditary form of the disease.

The Kaplan-Meier curves analysis showed that overall survival time was shorter for 55MM carriers compared to other genotype carriers with PC. About $14.4 \%$ less patients with 55MM than 55LM and 55LL survived 5 years from the time of PC diagnosis.

It seems also that carrying of $192 \mathrm{RR}$ is a good prognostic factor in relation to the overall survival time of PC men. All 192RR carriers survived at least 5 years from the moment of PC diagnosis (and even over 7.5 years).

The 192RR genotype probably may be associated with the age lower than 60 years at PC onset. It is worth noting that $192 \mathrm{RR}$ was detected in $6.9 \%$ of PC men compared to $1.9 \%$ of healthy men $<60$ years of age, however, this difference was not statistically significant ( $\mathrm{p}=0.24)$.

We suggest that the presence of 55MM homozygous genotype may be probably associated with an aggressive form of prostate cancer. The similar observations were made by Stevens et al. in the US population. They observed the 2.18-fold higher risk of aggressive PC (T3/4; GS $\geq 8$ ) in 192QR/55LM heterozygous haplotype carriers, compared to the population risk $(\mathrm{OR}=2.18 ; \mathrm{p} \leq 0.05)$. Authors suggested that men with PON1 L55M and Q192R genotypes that reduce paraoxonase 1 - mediated ROS detoxification capacity may have increased risk of aggressive prostate cancer [8].

Concluding, we suggest that PON1 55MM variant may be probably associated with younger age at PC onset in men from families with HPC and with a shorter survival. However, more extensive studies on a larger number of PC patients, possibly from various populations, are necessary to confirm, and extend our findings. Performing similar investigations on a larger group of PC patients, also in different populations, will be promising. PON1 polymorphisms usefulness as a biomarkers for screening, diagnosis and measurement of treatment efficacy with respect to prognosis and survival in prostate cancer patients should be determined.

This study was supported by the fund of the Department of Clinical Genetics of Collegium Medicum Nicolaus Copernicus University, Bydgoszcz, Poland.

The authors declare no conflict of interest.

\section{References}

1. Siegel RL, Miller KD, Jemal A. Cancer Statistics, 2017. CA Cancer J Clin 2017; 67: 7-30.

2. Li C, Long B, Qin X, et al. Cytochrome P1B1 (CYP1B1) polymorphisms and cancer risk: a meta-analysis of 52 studies. Toxicology 2015; 327: 77-86.

3. Arpaci A, Görmüs U, Dalan B, et al. Investigation of PON1 192 and PON1 55 polymorphisms in ovarian cancer patients in Turkish population. In Vivo 2009; 23: 421-424.

4. Wu J, Fang M, Zhou X, et al. Paraoxonase 1 gene polymorphisms are associated with an increased risk of breast cancer in a population of Chinese women. Oncotarget 2017; 8: 2536225371.

5. Wen Y, Huang Z, Zhang X, et al. Correlation between PON1 gene polymorphisms and breast cancer risk: a meta-analysis. Int J Clin Exp Med 2015; 8: 20343-20348.

6. Hu P, Ma Y, Zhang L, et al. PON1 L55M polymorphism might contribute to the risk of cancer. Panminerva Med 2017; 59: 107-113.

7. Chen L, Lu W, Fang L, et al. Association between L55M polymorphism in paraoxonase 1 and cancer risk: a meta-analysis based on 21 studies. Onco Targets Ther 2016; 9: 1151-1158.

8. Stevens VL, Rodriguez C, Talbot JT, et al. Paraoxonase 1 (PON1) polymorphisms and prostate cancer in the CPS-II Nutrition Cohort. Prostate 2008; 68: 1336-1340.

9. Wang H, Li L, Ding L, et al. Association of genetic polymorphisms in the paraoxonase 1 gene with the risk and prognosis of non-small cell lung cancer in Chinese Han population. J Investig Med 2012; 60: 592-597.

10. Eom SY, Yim DH, Lee CH, et al. Interactions between paraoxonase 1 genetic polymorphisms and smoking and their effects on oxidative stress and lung cancer risk in a Korean population. PLoS One 2015; 10: e0119100.

11. Fang DH, Fan CH, Ji Q, et al. Differential effects of paraoxonase 1 (PON1) polymorphisms on cancer risk: evidence from 25 published studies. Mol Biol Rep 2012; 39: 6801-6809.

12. Aldonza MB, Son YS, Sung HJ, et al. Paraoxonase-1 (PON1) induces metastatic potential and apoptosis escape via its antioxidative function in lung cancer cells. Oncotarget 2017; 8: 42817-42835.

13. Trachootham D, Lu W, Ogasawara MA, et al. Redox regulation of cell survival. Antioxid Redox Signal 2008; 10: 1343-1374.

14. Iwanicka J, Iwanicki T, Niemiec P, et al. Relationship between rs854560 PON1 gene polymorphism and tobacco smoking with coronary artery disease. Dis Markers 2017; 2017: 1540949 .

15. Tomatir AG, Pehlivan S, Sahin HH, et al. Q192R and L55M polymorphisms of paraoxonase 1 gene in chronic myelogenous leukemia and chronic lymphocytic leukemia. Anticancer Res 2015; 35: 4807-4812. 
16. Matés JM, Pérez-Gómez C, Blanca M. Chemical and biological activity of free radical 'scavengers' in allergic diseases. Clin Chim Acta 2000; 296: 1-15.

17. Lobo V, Patil A, Phatak A, et al. Free radicals, antioxidants and functional foods: Impact on human health. Pharmacogn Rev 2010; 4: 118-126.

18. Manolescu BN, Busu C, Badita D, et al. Paraoxonase 1 - an update of the antioxidant properties of high-density lipoproteins. Maedica (Buchar) 2015; 10: 173-177.

19. Kao YL, Donaghue K, Chan A, et al. A variant of paraoxonase (PON1) gene is associated with diabetic retinopathy in IDDM J. Clin Endocrinol Metab 1998; 83: 2589-2592.

20. El-Lebedy D, Kafoury M, Abd-El Haleem D, et al. Paraoxonase-1 gene Q192R and L55M polymorphisms and risk of cardiovascular disease in Egyptian patients with type 2 diabetes mellitus. J Diabetes Metab Disord 2014; 13: 124.

21. Zielaskowska J, Olszewska-Słonina D. The polymorphism of paraoxonase and its effects in physiological and pathological processes. Adv Clin Exp Med 2006; 15: 1073-1078.

22. Eras N, Tombak A, Tiftik N, et al. Association between PON1 L55M polymorphism and PON1 enzyme activity in patients with leukemia. Int J Hematol Oncol 2017; 1: 21-28.

23. Mackness B, Mackness MI, Arrol S, et al. Effect of the molecular polymorphisms of human paraoxonase (PON1) on the rate of hydrolysis of paraoxon. Br J Pharmacol 1997; 122: 265268.

24. Shunmoogam N, Naidoo P, Chilton R. Paraoxonase (PON)-1: a brief overview on genetics, structure, polymorphisms and clinical relevance. Vasc Health Risk Manag 2018; 14: 137-143.

25. Marchesani M, Hakkarainen A, Tuomainen TP, et al. New paraoxonase 1 polymorphism I102V and the risk of prostate cancer in Finnish men. J Natl Cancer Inst 2003; 95: 812-818

26. Carter BS, Bova GS, Beaty TH, et al. Hereditary prostate cancer: epidemiologic and clinical features. J Urol 1993; 150 797-802.

27. Cybulski C, Gliniewicz B, Sikorski A, et al. Hereditary prostate cancer: Clinical genetics of cancers 2018. Lubiński J (ed). Print Group Sp. z o.o., Poland 2018; 179-192.

28. Antognelli C, Mearini L, Talesa VN, et al. Clinical genetics of cancers. Prostate 2005; 63: 240-251.

29. Fang DH, Fan CH, Ji Q, et al. Differential effects of paraoxonase 1 (PON1) polymorphisms on cancer risk: evidence from 25 published studies. Mol Biol Rep 2012; 39: 6801-6809.

30. Zhang M, Xiong H, Fang L, et al. Paraoxonase 1 (PON1) Q192R gene polymorphism and cancer risk: A meta-analysis based on 30 publications. Asian Pac J Cancer Prev 2015; 16: 4457-4463.

31. Chen L, Lu W, Fang L, et al. Association between L55M polymorphism in paraoxonase 1 and cancer risk: a meta-analysis based on 21 studies. J Onco Targets Ther 2016; 9: 1151-1158.

\section{Address for correspondence}

\section{Marta Heise}

Department of Clinical Genetics

Collegium Medicum in Bydgoszcz

M. Curie-Skłodowskiej 9

85-094 Bydgoszcz, Poland

tel. 525853567

fax. 525853568

e-mail: marta.heise@onet.pl 